\title{
Preliminary antifungal and cytotoxic evaluation of synthetic cycloalkyl $[b]$ thiophene derivatives with PLS-DA analysis
}

BEATRIZ C. C. SOUZA ${ }^{1}$

TIAGO B. DE OLIVEIRA ${ }^{1}$

THIAGO M. AQUINO ${ }^{1}$

MARIA C. A. DE LIMA ${ }^{1}$

IVAN R. PITTA ${ }^{1}$

SUELY L. GALDINO ${ }^{1}$

EDELTRUDES O. LIMA²

TERESINHA GONÇALVES-SILVA ${ }^{1}$

GARDÊNIA C. G. MILITÃO ${ }^{1}$

LUCIANA SCOTTI ${ }^{2}$

MARCUS T. SCOTTI ${ }^{3}$

FRANCISCO J. B. MENDONÇA Jr. ${ }^{4}$

${ }^{1}$ Departamento de Antibióticos

Universidade Federal de Pernambuco

Recife-PE 50670-910,Brazil

2 Departamento de Ciências Farmacêuticas Universidade Federal da Paraíba João Pessoa-PB, 58051-970, Brazil

${ }^{3}$ Departamento de Engenharia e Meio Ambiente, Universidade Federal da Paraíba, Campus IV; Rio Tinto-PB 58297-000, Brazil

4 Departamento de Ciências Biológicas, Universidade Estadual da Paraíba CCBSA, João Pessoa-PB 58070-450, Brazil

\begin{abstract}
A series of 2-[(arylidene)amino]-cycloalkyl[ $b]$ thiophene-3-carbonitriles (2a-x) was synthesized by incorporation of substituted aromatic aldehydes in Gewald adducts (1a-c). The title compounds were screened for their antifungal activity against Candida krusei and Criptococcus neoformans and for their antiproliferative activity against a panel of 3 human cancer cell lines (HT29, NCI H-292 and HEP). For antiproliferative activity, the partial least squares (PLS) methodology was applied. Some of the prepared compounds exhibited promising antifungal and proliferative properties. The most active compounds for antifungal activity were cyclohexyl[ $b]$ thiophene derivatives, and for antiproliferative activity cycloheptyl[ $b]$ thiophene derivatives, especially 2 -[( $1 \mathrm{H}$-indol-2-yl-methylidene)amino]-5,6,7,8-tetrahydro- $4 H$-cyclohepta[ $b]$ thiophene-3-carbonitrile (2r), which inhibited more than $97 \%$ growth of the three cell lines. The PLS discriminant analysis (PLS-DA) applied generated good exploratory and predictive results and showed that the descriptors having shape characteristics were strongly correlated with the biological data.

Keywords: cycloalkyl[ $b]$ thiophene derivatives, antifungal activity, antiproliferative activity, PLS-DA, Pentacle program
\end{abstract}

2-Aminothiophene derivatives are an important class of heterocycles found in several biologically active and natural compounds. This class of compounds has demonstrated a broad spectrum of activities and applications as pharmaceuticals and agrochemicals, dyes, biodiagnostics, and electronic and optoelectronic devices (1).

* Correspondence; e-mail: franciscojbmendonca@yahoo.com.br 
B. C. C. Souza et al.: Preliminary antifungal and cytotoxic evaluation of synthetic cycloalkyl[b]thiophene derivatives with PLS-DA analysis, Acta Pharm. 62 (2012) 221-236.

Substituted 2-aminothiophenes are a class of heterocycles that have attracted a great deal of research interest due their great usefulness as precursors of molecules with pharmacological properties. They have been reported to exert antitubercular (2), anti-inflammatory (3), antimicrobial (4) and antianxiety (5) properties. A survey of the literature also reveals that substituted 2-aminothiophenes are potent and selective inhibitors of human leukocyte elastase (6), kinesin spindle protein (KPS) (7) and adenosine A1 receptor allosteric enhancers (8). Antifungal (9) and antitumor (10) properties have also been extensively described, resulting in marketed antifungal agents such as sertaconazole.

The above prompted us to synthesize a new series of 2-[(arylidene)amino]-cycloal$\operatorname{kyl}[b]$ thiophene-3-carbonitrile derivatives by incorporating different substituted aromatic aldehydes at the 2-amino position of the thiophene ring, thereby affording Schiff bases. These were screened for their in vitro antifungal and antiproliferative activities. Partial Least Squares discriminant analysis (PLS-DA) was applied, using Pentacle, to the antiproliferative activity of cycloalkyl $[b]$ thiophene derivatives.

\section{EXPERIMENTAL}

All melting points were measured on a Quimis-340.27 apparatus (Quimis, Brazil) and are uncorrected. IR spectra were recorded using potassium bromide pellets on a Bruker IFS-66 IR spectrophotometer (Bruker, USA). NMR were recorded on a Unity Plus-300 MHz-Varian spectrometer (Varian, USA) using tetramethylsilane as internal standard. Chemical shifts are reported in ppm $(\delta)$, and coupling constants $(J)$ are reported in Hz. HRMS were recorded on a Delsi-Nermag R1010C mass spectrometer (Delsi-Nermag, France) with $70 \mathrm{eV}$ electron impact. Elemental analyses were performed using an EA 1110 CHNS-O elemental analyzer (CE instruments, UK). The results were found to be in accord $( \pm 0.4 \%)$ with the calculated values. All reactions were monitored by TLC on $0.25 \mathrm{~mm}$ silica gel plates $\left(60 \mathrm{~F}_{254}\right.$, Merck, Germany) using binary mixtures of hexane/ethyl acetate in different proportions (from 9:1 to 1:1, $V / V$ ). Spectral, physical and analytical data of all newly synthesized compounds are listed in Tables I-III.

\section{General synthesis procedure}

2-[(Arylidene)amino]-cycloalkyl[ $b]$ thiophene-3-carbonitriles (2a-x). - An equimolar mixture of 1a-c and substituted aromatic aldehyde in ethanol with $0.5 \mathrm{~mL}$ of acetic acid was stirred under reflux for $2 \mathrm{~h}$ and then cooled to room temperature. Water was added and the solid that precipitated out was filtered under vacuum, washed with water, dried and recrystallized from absolute ethanol (Scheme 1).

\section{Antifungal activity}

The in vitro antifungal activity of synthesized compounds $\mathbf{2 a - 0 ,} \mathbf{2} \mathbf{r}-\mathbf{x}$ was investigatedfor two species of pathogenic fungi: Candida krusei LM08 and Criptococcus neoformans ICB59. These strains were supplied by the URM Culture Collection of the Department of Mycology, Department of Pharmaceutical Sciences of the Federal University of Paraíba, Brazil. Minimum inhibitory concentration (MIC) values were determined by the micro- 
B. C. C. Souza et al.: Preliminary antifungal and cytotoxic evaluation of synthetic cycloalkyl[ $b]$ thiophene derivatives with PLS-DA analysis, Acta Pharm. 62 (2012) 221-236.

\begin{tabular}{|c|c|c|c|}
\hline Compound & & $\mathrm{R}$ & $\mathrm{R}^{\prime}$ \\
\hline $1 a$ & 1 & - & - \\
\hline $1 \mathrm{~b}$ & 2 & - & - \\
\hline 1c & 3 & - & - \\
\hline $2 a$ & 1 & & $\mathrm{H}$ \\
\hline $2 b$ & 3 & & $\mathrm{H}$ \\
\hline $2 c$ & 1 & & $4-\mathrm{Cl}$ \\
\hline $2 d$ & 2 & & $4-\mathrm{Cl}$ \\
\hline $2 e$ & 3 & & $4-\mathrm{Cl}$ \\
\hline $2 f$ & 1 & & $4-\mathrm{Br}$ \\
\hline $2 g$ & 2 & & $4-\mathrm{Br}$ \\
\hline $2 \mathrm{~h}$ & 3 & & $4-\mathrm{Br}$ \\
\hline $2 i$ & 2 & & $4-\mathrm{F}$ \\
\hline $2 j$ & 2 & & $4-\mathrm{OCH}_{3}$ \\
\hline $2 k$ & 2 & & $4-O B z$ \\
\hline 21 & 3 & & $4-\mathrm{NO}_{2}$ \\
\hline $2 m$ & 3 & & $4-\mathrm{CH}_{3}$ \\
\hline $2 n$ & 3 & & $4-E t$ \\
\hline 20 & 3 & & 4-IPr \\
\hline $2 p$ & 2 & & - \\
\hline $2 q$ & 2 & & - \\
\hline $2 r$ & 3 & & - \\
\hline $2 \mathrm{~s}$ & 1 & & - \\
\hline $2 t$ & 2 & & - \\
\hline $2 u$ & 3 & & - \\
\hline $2 v$ & 1 & & - \\
\hline $2 w$ & 2 & & - \\
\hline $2 x$ & 3 & & - \\
\hline
\end{tabular}

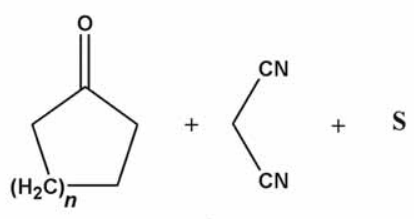

$\mathrm{Et}_{3} \mathrm{~N} \underset{\mathrm{rt}}{\mathrm{EtOH}}$

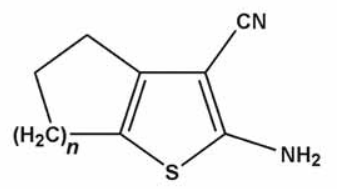

1a-c
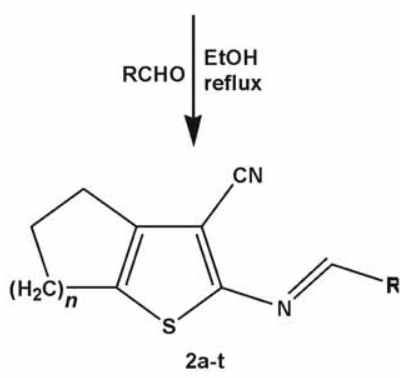

Scheme 1.

dilution broth method using microdilution plates according to the guidelines of the National Committee for Clinical and Laboratory Standards (NCCLS). The MIC for all strains was set at the lowest concentration of the antifungal agent that completely inhibited the growth of the organism, as detected by the naked eye when compared to the control group.

All the strains were stored in mineral oil at $18{ }^{\circ} \mathrm{C}$. The viability test and subsequent taxonomic confirmation were carried out according to Barnett et al. (12). In order to obtain an inoculum of $2.5 \times 10^{3}$ cells $\mathrm{mL}^{-1}$, each strain was cultured in a tube containing 20 $\mathrm{mL}$ of Sabouraud dextrose agar plus yeast extract at $35^{\circ} \mathrm{C}$ for two days. After this time, suspensions were prepared in a sterile physiological solution $(0.85 \%)$ and mixed in a shaker. The inoculum was adjusted to $90 \%$ transmittance at $530 \mathrm{~nm}$, as measured on a spectrophotometer. Stock solutions of tested compounds were freshly prepared in dimethyl sulfoxide (DMSO), which had no effect on the organism at the concentration studied, aliquoted, and stored at $-20{ }^{\circ} \mathrm{C}$ in stock solution at a final concentration of $1024 \mu \mathrm{g} \mathrm{mL}-1$. Decimal dilutions of thiophene stock solutions were prepared in free RPMI 1640 cell cul- 
B. C. C. Souza et al.: Preliminary antifungal and cytotoxic evaluation of synthetic cycloalkyl[ $b]$ thiophene derivatives with PLS-DA analysis, Acta Pharm. 62 (2012) 221-236.

ture medium (Sigma, USA) and buffered to $\mathrm{pH} 7.0$ with $0.165 \mathrm{~mol} \mathrm{~L}^{-1}$ of morpholinopropanesulphonic acid (MOPS, Sigma, USA). Miconazole and 5-fluorocitosine were used as reference drugs for anti-Candida and anti-Cryptococcus activity, respectively, and were tested under similar conditions. Microdilution plates containing serial dilutions (from 1024 to $1 \mu \mathrm{g} \mathrm{mL}-1$ ) of each compound were inoculated with each organism. Each plate included a positive control (fungi without any compound), negative control (medium only) and reference drugs. The microdilution plates were incubated at $35^{\circ} \mathrm{C}$ and were read visually after 24 and $72 \mathrm{~h}$ of incubation. All tests were performed in duplicate and the results were expressed as the arithmetic mean of MIC values obtained in the two trials (13). The bands established for antifungal activity were: excellent activity (50 to $500 \mu \mathrm{g}$ $\left.\mathrm{mL}^{-1}\right)$; moderate activity (600 to $\left.1000 \mu \mathrm{g} \mathrm{mL}^{-1}\right)$; inactive or poor activity $\left(1000 \mu \mathrm{g} \mathrm{mL}^{-1}\right)$ (14).

\section{Antiproliferative activity}

All synthesized compounds were selected by the National Cancer Institute (NCI) Developmental Therapeutic Program for in vitro cell line screening. Antiproliferative assays were performed according the US NCI protocol as described elsewhere (15). The compounds were evaluated in one primary dose $(25 \mu \mathrm{L} \mathrm{mL}-1)$ for three human cancer cell lines: colon carcinoma (HT29), lung cancer (NCI H-292) and laryngeal carcinoma (HEP) cells. The cells lines were supplied by the Rio de Janeiro Cell Bank of the Federal University of Rio de Janeiro (UFRJ), Brazil.

The cytotoxicity/survival of cells in the presence or absence of the experimental agent was determined using the MTT (3-[4,5-dimethylthiazol-2-yl]-2,5-diphenyltetrazolium bromide) method, as described previously (16). Cells harvested in the log phase of growth were counted and seeded in triplicate $\left(10^{5}\right.$ cells per $100 \mu \mathrm{L}$ per well $)$ in 96 -well microculture plates in a complete free Dulbecco's modified eagle's medium (DMEM). After $24 \mathrm{~h}$ of incubation at $37^{\circ} \mathrm{C}$ and $5 \% \mathrm{CO}_{2}$, the cultures were treated with the tested compounds at a single concentration of $25 \mu \mathrm{g} \mathrm{mL}-1$ in DMSO $(100 \mu \mathrm{L}$ per well). Each plate also included a positive control (cells without any compound) and a negative control (medium only). After $72 \mathrm{~h}$ of exposure to the compounds, $25 \mu \mathrm{L}$ of MTT was added to each well. After $4 \mathrm{~h}$ at $37^{\circ} \mathrm{C}$, the reaction was stopped and the formazan crystals formed by MTT metabolism were solubilized by additing $50 \mu \mathrm{L}$ of DMSO to each well. The cellular metabolism of MTT was quantified by reading the absorbance of the solubilized product at $595 \mathrm{~nm}$ with a 96-well plate reader attached to a spectrophotometer. Results for each tested compound were reported as the percentage growth of the treated cells compared to the untreated control cells. The mean \pm SD of three independent experiments for each compound was calculated. The compounds were classified as possessing no activity ( $0 \%$ growth inhibition), low activity (up to $30.0 \%$ growth inhibition), moderate activity (between $31.0 \%$ and $70.0 \%$ growth inhibition) and high activity $(>70.0 \%$ growth inhibition) for each cell line tested.

\section{Computational methods}

All compounds were drawn (2D structures) using ChemDraw 8.0. They were imported by the Spartan program for Windows 8.0 and were converted into 3D models. 
B. C. C. Souza et al.: Preliminary antifungal and cytotoxic evaluation of synthetic cycloalkyl[b]thiophene derivatives with PLS-DA analysis, Acta Pharm. 62 (2012) 221-236.

These models were minimized using the MM+ method (17) and atomic partial charges were assigned using the AM1 semiempirical method (18). These methods employed 1000 interactions, 100 cycles of optimization, and 10 conformers of lowest minimum energy. The selected dihedrals were evaluated by rotation in accordance with the standard (default) conditions of the program, in which the number of simultaneous variations was 1 to 8 . Acyclic chains were submitted to rotations from 60 to $180^{\circ}$ and torsion rings were in the range of 30 to $120^{\circ}$. Conformational search was applied and the $\mathrm{E}_{\mathrm{MIN}}$ conformer was selected and saved in sdf format (19).

The compounds were imported using the Pentacle program and the PLS methodology was applied. The Pentacle software, produced by Molecular Discovery (Italy), is a computational tool for computing alignment-free molecular descriptors, also called GRid-INDependent descriptors or GRIND (20). The software is based on Molecular Interaction Fields and describes the ability of the molecules to interact with other molecules and does not require superimposition of compounds. The Pentacle program uses the GRID force field to characterize potential polar and hydrophobic interaction sites around target molecules by water $\left(\mathrm{H}_{2} \mathrm{O}\right)$, hydrophobic (DRY) and carbonyl oxygen $(\mathrm{O})$ and amide nitrogen (N1) probe.

We calculated a binary free PLS-DA for the three cell lines investigated: HT29, NCI H-292 and HEP. The PLS-DA is a partial least squares regression of a set $(Y)$ of binary variables describing the categories of a category variable on a set $(X)$ of predictor variables. PLS-DA looks for components correlated with the unfolded class while describing a large number of variations in $\mathrm{X}$, i.e., directions able to discriminate between the classes. It is a compromise between the usual discriminant analysis and a discriminant analysis of the significant principal components of predictor variables. For the construction of binary PLS matrices the, compounds which showed cytotoxicity greater than $20 \%$ were considered active $=1$, and the compounds that did not reach $20 \%$ inactive $=0$.

\section{RESULTS AND DISCUSSION}

\section{Chemistry}

2-Amino-cycloalkyl[b]thiophene-3-carbonitriles (1a-c) were first synthesized by the reaction of malononitrile with a cyclic ketone and elemental sulphur in the presence of morpholine following the Gewald procedure $(9,10)$. Treatment of 1a-c with substituted aromatic aldehydes afforded 2-[(arylidene)amino]-cycloalkyl[ $b]$ thiophene-3-carbonitrile $(\mathbf{2 a}-\mathbf{x})$. The Schiff bases were prepared in satisfactory yield (except $\mathbf{2 q}$ ). Compounds $\mathbf{2 a - g}$ and $\mathbf{2} \mathbf{j}$ were also previously described in the literature (9).

Chemical structures of all newly synthesized compounds $(\mathbf{2 h}, \mathbf{2} \mathbf{i}$ and $\mathbf{2 k - x})$ were characterized on the basis of their physical, analytical and spectral data, and were in full agreement with the proposed structures (Tables I-III).

The main vibrational bands of the title compounds are given in Table III. IR spectra showed absorption bands at about 2960-2911 and 2223-2212 $\mathrm{cm}^{-1}$, characteristic of C-H and $\mathrm{C} \equiv \mathrm{N}$ stretching vibrations, respectively.

${ }^{1} \mathrm{H}$ NMR and ${ }^{13} \mathrm{C}$ NMR data of the compounds obtained are given in Table II and are consistent with the proposed structures. ${ }^{1} \mathrm{H}$ NMR spectra did not display signs of 2- 
B. C. C. Souza et al.: Preliminary antifungal and cytotoxic evaluation of synthetic cycloalkyl[ $b]$ thiophene derivatives with PLS-DA analysis, Acta Pharm. 62 (2012) 221-236.

Table I. Physical and analytical data of $2 h, i, k-x$

\begin{tabular}{ccccccc}
\hline \multirow{2}{*}{ Compd. } & $\begin{array}{c}\text { Molecular for- } \\
\text { mula }\end{array}$ & \multirow{2}{*}{$\begin{array}{c}\text { M. p. } \\
\left({ }^{\circ} \mathrm{C}\right)\end{array}$} & $\begin{array}{c}\text { Yield } \\
(\%)\end{array}$ & \multicolumn{2}{c}{ Analysis (calcd./found) $(\%)$} \\
\cline { 6 - 7 } & \multicolumn{1}{c}{$\left(M_{\mathrm{r}}\right)$} & & & $\mathrm{C}$ & $\mathrm{N}$ & $\mathrm{H}$ \\
\hline $\mathbf{2 h}$ & $\mathrm{C}_{17} \mathrm{H}_{15} \mathrm{BrN}_{2} \mathrm{~S}$ & 144 & 87 & $56.83 / 56.83$ & $7.80 / 7.77$ & $4.21 / 4.23$ \\
$\mathbf{2 i}$ & $\mathrm{C}_{16} \mathrm{H}_{13} \mathrm{FN}_{2} \mathrm{~S}$ & $93-95$ & 65 & $67.58 / 67.55$ & $9.85 / 9.85$ & $4.61 / 4.62$ \\
$\mathbf{2 k}$ & $\mathrm{C}_{23} \mathrm{H}_{20} \mathrm{~N}_{2} \mathrm{OS}$ & $140-142$ & 92 & $74.16 / 74.14$ & $7.52 / 7.50$ & $5.41 / 5.43$ \\
$\mathbf{2}$ & $\mathrm{C}_{17} \mathrm{H}_{15} \mathrm{~N}_{3} \mathrm{O}_{2} \mathrm{~S}$ & 126 & 90 & $62.75 / 62.71$ & $12.91 / 12.88$ & $4.65 / 4.66$ \\
$\mathbf{2 m}$ & $\mathrm{C}_{18} \mathrm{H}_{18} \mathrm{~N}_{2} \mathrm{~S}$ & $88-90$ & 92 & $73.43 / 73.44$ & $9.51 / 9.48$ & $6.16 / 6.19$ \\
$\mathbf{2 n}$ & $\mathrm{C}_{19} \mathrm{H}_{20} \mathrm{~N}_{2} \mathrm{~S}$ & $94-95$ & 84 & $73.99 / 73.98$ & $9.08 / 9.07$ & $6.54 / 6.54$ \\
$\mathbf{2 o}$ & $\mathrm{C}_{20} \mathrm{H}_{22} \mathrm{~N}_{2} \mathrm{~S}$ & $93-95$ & 70 & $74.49 / 74.48$ & $8.69 / 8.65$ & $6.88 / 6.91$ \\
$\mathbf{2 p}$ & $\mathrm{C}_{20} \mathrm{H}_{21} \mathrm{~N}_{3} \mathrm{OS}$ & $159-160$ & 74 & $68.35 / 68.34$ & $11.96 / 11.95$ & $6.02 / 6.03$ \\
$\mathbf{2 q}$ & $\mathrm{C}_{20} \mathrm{H}_{21} \mathrm{~N}_{3} \mathrm{~S}$ & 211 & 18 & $71.61 / 71.61$ & $12.53 / 12.51$ & $6.31 / 6.34$ \\
$\mathbf{2 r}$ & $\mathrm{C}_{19} \mathrm{H}_{17} \mathrm{~N}_{3} \mathrm{~S}$ & $180-182$ & 90 & $71.44 / 71.40$ & $13.15 / 13.12$ & $5.36 / 5.40$ \\
$\mathbf{2 s}$ & $\mathrm{C}_{18} \mathrm{H}_{13} \mathrm{~N}_{3} \mathrm{~S}$ & $200-202$ & 67 & $71.26 / 71.25$ & $13.85 / 13.85$ & $4.32 / 4.33$ \\
$\mathbf{2 t}$ & $\mathrm{C}_{19} \mathrm{H}_{15} \mathrm{~N}_{3} \mathrm{~S}$ & $175-177$ & 53 & $71.90 / 71.87$ & $13.24 / 13.21$ & $4.76 / 4.80$ \\
$\mathbf{2 u}$ & $\mathrm{C}_{20} \mathrm{H}_{17} \mathrm{~N}_{3} \mathrm{~S}$ & $204-206$ & 79 & $72.48 / 72.44$ & $12.68 / 12.67$ & $5.17 / 5.19$ \\
$\mathbf{2 v}$ & $\mathrm{C}_{13} \mathrm{H}_{10} \mathrm{~N}_{2} \mathrm{~S}_{2}$ & $174-175$ & 81 & $60.43 / 60.41$ & $10.84 / 10.80$ & $3.90 / 3.92$ \\
$\mathbf{2 w}$ & $\mathrm{C}_{14} \mathrm{H}_{12} \mathrm{~N}_{2} \mathrm{~S}_{2}$ & $123-125$ & 88 & $61.73 / 61.70$ & $10.28 / 10.25$ & $4.44 / 4.46$ \\
$\mathbf{2 x}$ & $\mathrm{C}_{15} \mathrm{H}_{14} \mathrm{~N}_{2} \mathrm{~S}_{2}$ & $115-117$ & 76 & $62.90 / 62.89$ & $9.78 / 9.77$ & $4.93 / 4.95$ \\
\hline
\end{tabular}

-aminothiophene protons $\left(\mathrm{NH}_{2}\right)$, but rather $\mathrm{CH}=\mathrm{N}$ protons, highlighting the success of the final synthetic step in obtaining the Schiff bases. Signals of the $\mathrm{CH}=\mathrm{N}$ protons were observed as a singlet in the $8.02-8.95$ ppm region, almost always as the stronger deshielding signal.

The strongest shielding signals and peaks in ${ }^{1} \mathrm{H}$ NMR and ${ }^{13} \mathrm{C}$ NMR were attributed to cycloalkyl protons and carbons. These appeared at $1.62-2.99 \mathrm{ppm}$ in ${ }^{1} \mathrm{H} \mathrm{NMR}$, and at 21.8-32.0 ppm in ${ }^{13} \mathrm{C}$ NMR. Further support was obtained from the ${ }^{13} \mathrm{C}$ NMR spectra, which exhibited resonance at $114.0-115.9 \mathrm{ppm}$ attributed to the $\mathrm{C} \equiv \mathrm{N}$ group.

The HRMS of all synthesized compounds exhibited $(\mathrm{M}+\mathrm{H})^{+}$molecular ion peaks, which is in agreement with the molecular formulas. For compounds $\mathbf{2 v}$ and $\mathbf{2 x}$, the molecular ion peaks found were $[\mathrm{M}+\mathrm{Na}]^{+}$.

\section{Antifungal activity}

All synthesized compounds (except $\mathbf{2 p}$ and $\mathbf{2 q}$ ) were evaluated for their in vitro antifungal activity against Criptococcus neoformans and Candida krusei. The data presented in Table IV show that the tested compounds more efficiently inhibited the growth of $C$. neoformans than the $C$. krusei strain. The MIC values ranged from excellent $\left(50-500 \mu \mathrm{g} \mathrm{mL}^{-1}\right)$ to moderate $\left(600-1500 \mu \mathrm{g} \mathrm{mL}^{-1}\right)$ for half of the tested compounds, although none exhibited greater antifungal activity than the reference drugs 5-fluorocytosine and miconazole. 
B. C. C. Souza et al.: Preliminary antifungal and cytotoxic evaluation of synthetic cycloalkyl[b]thiophene derivatives with PLS-DA analysis, Acta Pharm. 62 (2012) 221-236.

Table II. NMR data for $2 h, i, k-x$

\begin{tabular}{|c|c|c|}
\hline Compd. & ${ }^{1} \mathrm{H}$ NMR $(\delta \mathrm{ppm})^{\mathrm{a}}$ & ${ }^{13} \mathrm{C} \operatorname{NMR}(\delta \mathrm{ppm})^{\mathrm{a}}$ \\
\hline $2 \mathrm{~h}$ & $\begin{array}{l}1.64-1.74\left(\mathrm{~m}, 4 \mathrm{H}, \mathrm{CH}_{2}\right), 1.84-1.88\left(\mathrm{~m}, 2 \mathrm{H}, \mathrm{CH}_{2}\right) \\
2.76-2.81\left(\mathrm{~m}, 4 \mathrm{H}, \mathrm{CH}_{2}\right), 7.58(\mathrm{~d}, 2 \mathrm{H}, J=8.7 \mathrm{~Hz} \\
\mathrm{Ar}-\mathrm{H}), 7.78(\mathrm{~d}, 2 \mathrm{H}, J=8.7 \mathrm{~Hz}, \mathrm{Ar}-\mathrm{H}), 8.34(\mathrm{~s}, 1 \mathrm{H}, \\
\mathrm{CH}=\mathrm{N})\end{array}$ & $\begin{array}{l}\text { 157.1, 140.3, 136.7, 133.9, 132.1, } \\
\text { 130.5, 126.8, 114.9, 109.7, 31.9, } \\
30.6,29.1,27.7,27.0\end{array}$ \\
\hline $2 \mathrm{i}$ & $\begin{array}{l}1.84-1.90\left(\mathrm{~m}, 4 \mathrm{H}, \mathrm{CH}_{2}\right), 2.63-2.70\left(\mathrm{~m}, 4 \mathrm{H}, \mathrm{CH}_{2}\right) \\
7.12(\mathrm{~d}, 2 \mathrm{H}, J=8.7 \mathrm{~Hz}, \mathrm{Ar}-\mathrm{H}), 7.91(\mathrm{~d}, 2 \mathrm{H}, J=8.7 \\
\mathrm{Hz}, \mathrm{Ar}-\mathrm{H}), 8.36(\mathrm{~s}, 1 \mathrm{H}, \mathrm{CH}=\mathrm{N})\end{array}$ & $\begin{array}{l}\text { 166.8, 163.5, 159.4, 157.3, 135.1, } \\
\text { 132.5, 131.5, 131.4, 116.2, 115.9, } \\
\text { 114.4, 107.0, 25.1, 24.2, 23.0, } \\
21.9\end{array}$ \\
\hline $2 k$ & $\begin{array}{l}1.73-1.89\left(\mathrm{~m}, 4 \mathrm{H}, \mathrm{CH}_{2}\right), 2.62-2.69\left(\mathrm{~m}, 4 \mathrm{H}, \mathrm{CH}_{2}\right) \\
5.12(\mathrm{~s}, 2 \mathrm{H}, \mathrm{OCH}), 7.03(\mathrm{~d}, 2 \mathrm{H}, J=8.7 \mathrm{~Hz}, \mathrm{Ar}-\mathrm{H}), \\
7.34-7.46(\mathrm{~m}, 5 \mathrm{H}, \mathrm{Ar}-\mathrm{H}), 7.87(\mathrm{~d}, 2 \mathrm{H}, J=8.7 \mathrm{~Hz}, \\
\text { Ar-H), } 8.32 \\
(\mathrm{~s}, 1 \mathrm{H}, \mathrm{CH}=\mathrm{N})\end{array}$ & $\begin{array}{l}\text { 162.1, 160.4, 158.2, 136.0, 134.7, } \\
\text { 131.5, 131.3, 128.6, 128.1, 128.1, } \\
\text { 127.4, 115.1, 114.6, 105.8, 70.0, } \\
\text { 25.0, 24.2, 23.0, 21.9 }\end{array}$ \\
\hline 21 & $\begin{array}{l}1.66-1.75\left(\mathrm{~m}, 4 \mathrm{H}, \mathrm{CH}_{2}\right), 1.85-1.89\left(\mathrm{~m}, 2 \mathrm{H}, \mathrm{CH}_{2}\right) \\
2.79-2.82\left(\mathrm{~m}, 4 \mathrm{H}, \mathrm{CH}_{2}\right), 8.06(\mathrm{~d}, 2 \mathrm{H}, J=8.7 \mathrm{~Hz} \\
\mathrm{Ar}-\mathrm{H}), 8.28(\mathrm{~d}, 2 \mathrm{H}, J=8.7 \mathrm{~Hz}, \mathrm{Ar}-\mathrm{H}), 8.43(\mathrm{~s}, 1 \mathrm{H}, \\
\mathrm{CH}=\mathrm{N})\end{array}$ & $\begin{array}{l}155.8,155.0,149.3,140.9,140.4, \\
138.6,129.6,123.9,114.5,111.4 \\
31.8,30.7,29.0,27.6,26.9\end{array}$ \\
\hline $2 \mathrm{~m}$ & $\begin{array}{l}1.69\left(\mathrm{~m}, 4 \mathrm{H}, \mathrm{CH}_{2}\right), 1.86\left(\mathrm{~m}, 2 \mathrm{H}, \mathrm{CH}_{2}\right), 2.41(\mathrm{~s}, 3 \mathrm{H}, \\
\left.\mathrm{CH}_{3}\right), 2.78\left(\mathrm{~m}, 4 \mathrm{H}, \mathrm{CH}_{2}\right), 7.25(\mathrm{~d}, 2 \mathrm{H}, J=8.1 \mathrm{~Hz}, \\
\mathrm{Ar}-\mathrm{H}), 7.81(\mathrm{~d}, 2 \mathrm{H}, J=8.1 \mathrm{~Hz}, \mathrm{Ar}-\mathrm{H}), 8.38(\mathrm{~s}, 1 \mathrm{H}, \\
\mathrm{CH}=\mathrm{N})\end{array}$ & $\begin{array}{l}158.8,158.1,143.0,139.9,135.7 \\
132.5,129.5,129.3,115.0,108.7 \\
31.9,30.5,29.1,27.7,27.1,21.7\end{array}$ \\
\hline $2 n$ & $\begin{array}{l}1.26\left(\mathrm{t}, 3 \mathrm{H}, J=7.5 \mathrm{~Hz}, \mathrm{CH}_{3}\right), 1.64-1.74\left(\mathrm{~m}, 4 \mathrm{H}, \mathrm{CH}_{2}\right), \\
1.84-1.88\left(\mathrm{~m}, 2 \mathrm{H}, \mathrm{CH}_{2}\right), 2.73(\mathrm{q}, 2 \mathrm{H}, J=7.5 \mathrm{~Hz}, \\
\left.\mathrm{CH}_{2}\right), 2.75-2.81\left(\mathrm{~m}, 4 \mathrm{H}, \mathrm{CH}_{2}\right), 7.28(\mathrm{~d}, 2 \mathrm{H}, J=8.1 \\
\mathrm{Hz}, \mathrm{Ar}-\mathrm{H}), 7.84(\mathrm{~d}, 2 \mathrm{H}, J=8.1 \mathrm{~Hz}, \mathrm{Ar}-\mathrm{H}), 8.39(\mathrm{~s}, \\
1 \mathrm{H}, \mathrm{CH}=\mathrm{N})\end{array}$ & $\begin{array}{l}158.8,158.1,149.3,139.9,135.7, \\
132.7,129.5,128.4,115.0,108.7, \\
31.9,30.5,29.1,29.0,27.8,27.1 \\
15.2\end{array}$ \\
\hline 20 & $\begin{array}{l}1.72\left(\mathrm{~d}, 6 \mathrm{H}, J=6.9 \mathrm{~Hz}, \mathrm{CH}_{3}\right), 1.63-1.73(\mathrm{~m}, 4 \mathrm{H}, \\
\left.\mathrm{CH}_{2}\right), 1.83-1.91\left(\mathrm{~m}, 2 \mathrm{H}, \mathrm{CH}_{2}\right), 2.75-2.81(\mathrm{~m}, 4 \mathrm{H}, \\
\left.\mathrm{CH}_{2}\right), 2.96(\mathrm{~d}, 1 \mathrm{H}, J=6.9 \mathrm{~Hz}, \mathrm{CH}), 7.31(\mathrm{~d}, 2 \mathrm{H}, \\
J=8.1 \mathrm{~Hz}, \mathrm{Ar}-\mathrm{H}), 7.84(\mathrm{~d}, 2 \mathrm{H}, J=8.1 \mathrm{~Hz}, \mathrm{Ar}-\mathrm{H}), \\
8.38(\mathrm{~s}, 1 \mathrm{H}, \mathrm{CH}=\mathrm{N})\end{array}$ & $\begin{array}{l}\text { 158.7, 158.1, 153.8, 139.9, 135.6, } \\
\text { 132.8, 129.4, 126.9, 115.0, 108.7, } \\
34.2,31.9,30.5,29.0,27.7,27.0, \\
23.6\end{array}$ \\
\hline $2 p$ & $\begin{array}{l}1.80-1.88\left(\mathrm{~m}, 4 \mathrm{H}, \mathrm{CH}_{2}\right), 2.61-2.67\left(\mathrm{~m}, 4 \mathrm{H}, \mathrm{CH}_{2}\right), \\
3.30 \\
\left(\mathrm{t}, 4 \mathrm{H}, J=4.8 \mathrm{~Hz}, \mathrm{NCH}_{2}\right), 3.84(\mathrm{t}, 4 \mathrm{H}, J=4.8 \mathrm{~Hz}, \\
\left.\mathrm{OCH}_{2}\right), 6.88(\mathrm{~d}, 2 \mathrm{H}, J=9 \mathrm{~Hz}, \mathrm{Ar}-\mathrm{H}), 7.80(\mathrm{~d}, 2 \mathrm{H}, \\
J=9 \mathrm{~Hz}, \mathrm{Ar}-\mathrm{H}), 8.26(\mathrm{~s}, 1 \mathrm{H}, \mathrm{CH}=\mathrm{N})\end{array}$ & $\begin{array}{l}\text { 161.1, 158.3, 153.7, 134.5, 131.1, } \\
130.8,125.6,114.9,113.9,105.0 \\
66.4,47.4,25.0,24.2,23.0,21.9\end{array}$ \\
\hline $2 q$ & $\begin{array}{l}1.85\left(\mathrm{~m}, 4 \mathrm{H}, \mathrm{CH}_{2}\right), 2.07\left(\mathrm{~m}, 4 \mathrm{H}, \mathrm{CH}_{2}\right), 2.66(\mathrm{~m}, 4 \mathrm{H}, \\
\left.\mathrm{CH}_{2}\right), 3.43\left(\mathrm{~m}, 4 \mathrm{H}, \mathrm{CH}_{2}\right), 6.62(\mathrm{~d}, 2 \mathrm{H}, J=8.4 \mathrm{~Hz}, \\
\mathrm{Ar}-\mathrm{H}), 7.94(\mathrm{~d}, 2 \mathrm{H}, J=8.4 \mathrm{~Hz}, \mathrm{Ar}-\mathrm{H}), 8.30(\mathrm{~s}, 1 \mathrm{H}, \\
\mathrm{CH}=\mathrm{N})\end{array}$ & $\begin{array}{l}\text { 198.3, 190.2, 158.3, 151.6, 134.5, } \\
\text { 133.1, 115.0, 112.3, 111.2, 102.8, } \\
\text { 48.0, 47.6, 25.4, 25.3, 24.9, 24.3, } \\
\text { 23.1, 22.0 }\end{array}$ \\
\hline $2 r$ & $\begin{array}{l}1.65-1.72\left(\mathrm{~m}, 4 \mathrm{H}, \mathrm{CH}_{2}\right), 1.84-1.88\left(\mathrm{~m}, 2 \mathrm{H}, \mathrm{CH}_{2}\right) \\
2.75-2.82\left(\mathrm{~m}, 4 \mathrm{H}, \mathrm{CH}_{2}\right), 7.29-7.42(\mathrm{~m}, 4 \mathrm{H}, \mathrm{Ar}-\mathrm{H}) \\
7.67(\mathrm{~d}, 1 \mathrm{H}, J=3 \mathrm{~Hz}, \mathrm{Ar}-\mathrm{H}), 8.60(\mathrm{~s}, 1 \mathrm{H}, \mathrm{CH}=\mathrm{N})\end{array}$ & $\begin{array}{l}\text { 160.5, 153.4, 139.3, 136.9, 133.5, } \\
\text { 132.1, 124.8, 124.2, 123.0, 122.7, } \\
\text { 116.0, 115.7, 111.3, 106.7, 32.0, } \\
30.5,29.1,27.9,27.2\end{array}$ \\
\hline
\end{tabular}


B. C. C. Souza et al.: Preliminary antifungal and cytotoxic evaluation of synthetic cycloalkyl[ $b]$ thiophene derivatives with PLS-DA analysis, Acta Pharm. 62 (2012) 221-236.

Table II. continued

2s $\quad 2.48\left(\mathrm{q}, 2 \mathrm{H}, J=7.6 \mathrm{~Hz}, \mathrm{CH}_{2}\right), 2.91(\mathrm{t}, 2 \mathrm{H}, J=7.6 \mathrm{~Hz}, 163.5,154.3,150.0,149.0,145.3$, $\left.\mathrm{CH}_{2}\right), 2.99\left(\mathrm{t}, 2 \mathrm{H}, \mathrm{J}=7.6 \mathrm{~Hz}, \mathrm{CH}_{2}\right), 7.79(\mathrm{t}, 1 \mathrm{H}, \quad 140.1,137.1,130.2,129.7,128.3$, $J=7.6 \mathrm{~Hz}, \mathrm{Ar}-\mathrm{H}), 7.87(\mathrm{t}, 1 \mathrm{H}, J=7.6 \mathrm{~Hz}, \mathrm{Ar}-\mathrm{H})$, $125.2,123.9,122.1,114.3,104.7$, $8.08(\mathrm{~d}, 1 \mathrm{H}, J=4.8 \mathrm{~Hz}, \mathrm{Ar}-\mathrm{H}), 8.40(\mathrm{~d}, 1 \mathrm{H}, J=8.4$ $30.2,28.0,27.3$ $\mathrm{Hz}, \mathrm{Ar}-\mathrm{H}), 8.95(\mathrm{~d}, 1 \mathrm{H}, J=8.4 \mathrm{~Hz}, \mathrm{Ar}-\mathrm{H}), 8.02(\mathrm{~s}$, $1 \mathrm{H}, \mathrm{CH}=\mathrm{N}), 9.03(\mathrm{~d}, 1 \mathrm{H}, J=4.8 \mathrm{~Hz}, \mathrm{Ar}-\mathrm{H})$

2t $\quad 1.82-1.88\left(\mathrm{~m}, 4 \mathrm{H}, \mathrm{CH}_{2}\right), 2.65-2.74\left(\mathrm{~m}, 4 \mathrm{H}, \mathrm{CH}_{2}\right)$, $158.4,155.5,150.0,149.0,137.1$ $7.91(\mathrm{~d}, 2 \mathrm{H}, J=6 \mathrm{~Hz}, \mathrm{Ar}-\mathrm{H}), 8.90(\mathrm{t}, 2 \mathrm{H}, J=11.2 \mathrm{~Hz}, 135.8,134.8,130.2,129.7,128.2$, Ar-H), $8.95(\mathrm{~s}, 1 \mathrm{H}, \mathrm{CH}=\mathrm{N}), 9.02(\mathrm{~d}, 2 \mathrm{H}, \mathrm{J}=6 \mathrm{~Hz}$, Ar-H) $125.2,123.9,122.1,114.0,109.2$ $25.3,24.2,22.9,21.8$

$2 \mathrm{u} \quad 1.69-1.72\left(\mathrm{~m}, 4 \mathrm{H}, \mathrm{CH}_{2}\right), 1.88\left(\mathrm{~m}, 2 \mathrm{H}, \mathrm{CH}_{2}\right), 2.79-2.82$ 156.3, 155.1, 149.8, 148.8, 140.8, $\left(\mathrm{m}, 4 \mathrm{H}, \mathrm{CH}_{2}\right), 7.66-7.79(\mathrm{~m}, 2 \mathrm{H}, \mathrm{Ar}-\mathrm{H}), 7.84(\mathrm{~d}, 1 \mathrm{H}, 138.7,137.2,130.0,129.7,128.2$, $J=8.4 \mathrm{~Hz}, \mathrm{Ar}-\mathrm{H}), 8.17(\mathrm{~d}, 1 \mathrm{H}, J=4.5 \mathrm{~Hz}, \mathrm{Ar}-\mathrm{H})$, $125.2,123.9,122.0,114.5,111.4$ $8.90(\mathrm{~d}, 1 \mathrm{H}, \mathrm{J}=8.4 \mathrm{~Hz}, \mathrm{Ar}-\mathrm{H}), 8.93(\mathrm{~s}, 1 \mathrm{H}, \mathrm{CH}=\mathrm{N}), \quad 31.8,30.7,29.0,27.6,26.9$ $9.00(\mathrm{~d}, 1 \mathrm{H}, J=4.5 \mathrm{~Hz}, \mathrm{Ar}-\mathrm{H})$

2v $\quad 2.36-2.45\left(\mathrm{~m}, 2 \mathrm{H}, \mathrm{CH}_{2}\right), 2.80-2.92\left(\mathrm{~m}, 4 \mathrm{H}, \mathrm{CH}_{2}\right)$, $6.81(\mathrm{dd}, 1 \mathrm{H}, J=1.5,3.6 \mathrm{~Hz}, \mathrm{Ar}-\mathrm{H}), 7.11(\mathrm{~d}, 1 \mathrm{H}$, $J=3.6 \mathrm{~Hz}, \mathrm{Ar}-\mathrm{H}), 7.64(\mathrm{~d}, 1 \mathrm{H}, J=1.5 \mathrm{~Hz}, \mathrm{Ar}-\mathrm{H})$, $8.25(\mathrm{~s}, 1 \mathrm{H}, \mathrm{CH}=\mathrm{N})$

2w $\quad 1.82-1.91\left(\mathrm{~m}, 4 \mathrm{H}, \mathrm{CH}_{2}\right), 2.63-2.69\left(\mathrm{~m}, 4 \mathrm{H}, \mathrm{CH}_{2}\right)$, $6.58(\mathrm{dd}, 1 \mathrm{H}, J=1.5,3.3 \mathrm{~Hz}, \mathrm{Ar}-\mathrm{H}), 7.11(\mathrm{~d}, 1 \mathrm{H}$, $J=3.3 \mathrm{~Hz}, \mathrm{Ar}-\mathrm{H}), 7.65(\mathrm{~d}, 1 \mathrm{H}, J=1.5 \mathrm{~Hz}, \mathrm{Ar}-\mathrm{H})$, $8.23(\mathrm{~s}, 1 \mathrm{H}, \mathrm{CH}=\mathrm{N})$

$164.9,151.2,146.9,145.2,144.8$, $137.4,118.0,114.7,112.9,101.6$, $30.1,28.1,27.2$

$159.7,151.3,146.9,146.3,135.3$, $132.3,117.8,114.3,112.9,106.1$, $25.1,24.2,22.9,21.9$

$2 x$ 1.62-1.72 (m, 4H, $\left.\mathrm{CH}_{2}\right), 1.82-1.86\left(\mathrm{~m}, 2 \mathrm{H}, \mathrm{CH}_{2}\right)$,

$157.7,151.2,146.8,146.1,140.3$, 2.74-2.79 (m, 4H, $\left.\mathrm{CH}_{2}\right), 6.57(\mathrm{dd}, 1 \mathrm{H}, \mathrm{J}=1.5,3.6 \mathrm{~Hz}, 136.0,117.7,114.9,112.8,108.4$, Ar-H), $7.10(\mathrm{~d}, 1 \mathrm{H}, \mathrm{J}=3.6 \mathrm{~Hz}, \mathrm{Ar}-\mathrm{H}), 7.64(\mathrm{~d}, 1 \mathrm{H}, \mathrm{J}$ 31.9, 30.4, 29.1, 27.7, 27.0 $=1.5 \mathrm{~Hz}, \mathrm{Ar}-\mathrm{H}), 8.23(\mathrm{~s}, 1 \mathrm{H}, \mathrm{CH}=\mathrm{N})$

a $\mathrm{CDCl}_{3}$

Compounds $2 \mathbf{t}$ and $\mathbf{2} \mathbf{u}$, with MIC values of $64 \mu \mathrm{g} \mathrm{mL}-1$, and compounds $\mathbf{2} \mathbf{d}, \mathbf{2} \mathbf{g}, \mathbf{2} \mathbf{j}$, $\mathbf{2 k}, \mathbf{2 m}, \mathbf{2 o}$ and $\mathbf{2 v}$ with MIC values of $128 \mu \mathrm{g} \mathrm{mL}^{-1}$, exhibited excellent antifungal activity against Criptococcus neoformans. Other tested compounds were inactive, or showed moderate activity (2a). The most active compounds for anti-Cryptococcus activity were $\mathbf{2 t}$ and $2 \mathbf{u}$, which have in common the presence of a quinoline moiety linked to the 2-amino position of the thiophene ring. This result corroborates the data of Boateng and collaborators (21) for a series of benzothieno[3,2-b]quinoline derivatives.

The compounds showed moderate activity against $C$. krusei. Compounds $\mathbf{2} \mathbf{i}, \mathbf{2 k}$ and $\mathbf{2 t}$ were the most active $(M I C=512 \mu \mathrm{g} \mathrm{mL}-1)$, followed by compounds $2 \mathbf{a}, \mathbf{2} \mathbf{g}, \mathbf{2} \mathbf{j}, \mathbf{2} \mathbf{r}$ and 2s (MIC values at $1024 \mu \mathrm{g} \mathrm{mL}^{-1}$ ).

The present study also confirmed that the presence of the cyclohexyl ring linked to the C-4 and C-5 position of the thiophene ring, as found in compounds $2 \mathbf{d}, 2 \mathbf{g}, 2 \mathbf{i}-\mathbf{k}$ and $2 t$, increases antifungal activity. The cyclopentyl (2a, 2s and $2 v$ ) and cycloheptyl (2l-o and 2r) derivatives displayed similar antifungal activity profiles and were, generally, two to four times less active than the cyclohexyl derivatives (9). 
B. C. C. Souza et al.: Preliminary antifungal and cytotoxic evaluation of synthetic cycloalkyl[b]thiophene derivatives with PLS-DA analysis, Acta Pharm. 62 (2012) 221-236.

Table III. HRMS and IR data for $2 h, i, k-x$

\begin{tabular}{|c|c|c|c|}
\hline \multirow{2}{*}{ Compd. } & \multirow{2}{*}{$\begin{array}{c}\text { IR }(\mathrm{KBr}) \\
\left(v_{\max } \mathrm{cm}^{-1}\right)\end{array}$} & \multicolumn{2}{|c|}{ HRMS $m / z(E S+)$} \\
\hline & & Calculated & Found \\
\hline $2 \mathrm{~h}$ & $\begin{array}{l}\text { CH (2916) } \\
\text { CN (2223) }\end{array}$ & 358.0139 & 359.0940 \\
\hline $2 \mathrm{i}$ & $\begin{array}{l}\mathrm{CH}(2940) \\
\mathrm{CN}(2222)\end{array}$ & 284.0783 & 285.0903 \\
\hline $2 k$ & $\begin{array}{l}\mathrm{CH}(2938) \\
\mathrm{CN}(2218)\end{array}$ & 372.1296 & 373.1119 \\
\hline 21 & $\begin{array}{l}\mathrm{CH}(2929) \\
\mathrm{CN}(2220)\end{array}$ & 325.0884 & 326.0718 \\
\hline $2 \mathrm{~m}$ & $\begin{array}{l}\mathrm{CH}(2912) \\
\mathrm{CN}(2222)\end{array}$ & 294.1190 & 295.1067 \\
\hline $2 n$ & $\begin{array}{l}\mathrm{CH}(2928) \\
\mathrm{CN}(2216)\end{array}$ & 308.1347 & 309.1455 \\
\hline 20 & $\begin{array}{l}\mathrm{CH}(2927) \\
\mathrm{CN}(2216)\end{array}$ & 322.1503 & 323.1641 \\
\hline $2 p$ & $\begin{array}{l}\mathrm{CH}(2945) \\
\mathrm{CN}(2213)\end{array}$ & 351.1405 & 352.1217 \\
\hline $2 q$ & $\begin{array}{l}\mathrm{CH}(2928) \\
\mathrm{CN}(2212)\end{array}$ & 335.1456 & 336.1291 \\
\hline $2 r$ & $\begin{array}{l}\mathrm{CH}(2911) \\
\mathrm{CN}(2220)\end{array}$ & 319.1143 & 320.1003 \\
\hline $2 s$ & $\begin{array}{l}\mathrm{CH}(2924) \\
\mathrm{CN}(2220)\end{array}$ & 303.0830 & 304.0767 \\
\hline $2 t$ & $\begin{array}{l}\mathrm{CH}(2937) \\
\mathrm{CN}(2217)\end{array}$ & 317.0986 & 318.1099 \\
\hline $2 u$ & $\begin{array}{l}\mathrm{CH}(2930) \\
\mathrm{CN}(2219)\end{array}$ & 331.1143 & 332.1270 \\
\hline $2 v$ & $\begin{array}{l}\mathrm{CH}(2960) \\
\mathrm{CN}(2220)\end{array}$ & 258.0285 & $281.0148^{\mathrm{a}}$ \\
\hline $2 w$ & $\begin{array}{l}\text { CH (2936) } \\
\text { CN (2213) }\end{array}$ & 272.0441 & 273.0399 \\
\hline $2 x$ & $\begin{array}{l}\mathrm{CH}(2929) \\
\mathrm{CN}(2215)\end{array}$ & 286.0598 & $309.0227^{\mathrm{a}}$ \\
\hline
\end{tabular}

$\mathrm{a}[\mathrm{M}+\mathrm{Na}]^{+}$

\section{Antiproliferative activity}

Table V summarizes the in vitro antiproliferative effects of cycloalkyl[ $b]$ thiophene derivatives $\mathbf{2 a - x}$ against the three human cancer cell lines. Compounds $\mathbf{2 a}, \mathbf{2 c}, \mathbf{2} \mathbf{f}-\mathbf{g}, \mathbf{2 k}-\mathbf{1}$, $2 p-q$ and $2 s-v$ exhibited antiproliferative activity below the concentration of $25 \mu \mathrm{g} \mathrm{mL}-1$.

The results indicate that the size of the cycloalkyl ring coupled at the C-4 and C-5 position of the thiophene ring is essential for antiproliferative activity. Increasing the si- 
B. C. C. Souza et al.: Preliminary antifungal and cytotoxic evaluation of synthetic cycloalkyl[ $b]$ thiophene derivatives with PLS-DA analysis, Acta Pharm. 62 (2012) 221-236.

Table IV. Antifungal activity of the synthesized compounds, miconazole and 5-fluorocitosine

\begin{tabular}{|c|c|c|}
\hline \multirow{2}{*}{ Compd. ${ }^{a}$} & \multicolumn{2}{|c|}{ MIC $\left(\mu \mathrm{g} \mathrm{mL}^{-1}\right)$} \\
\hline & C. neoformans & C. krusei \\
\hline $2 a$ & 512 & 1024 \\
\hline $2 b$ & - & - \\
\hline $2 c$ & - & - \\
\hline $2 d$ & 128 & - \\
\hline $2 e$ & - & - \\
\hline $2 f$ & - & - \\
\hline $2 g$ & 128 & 1024 \\
\hline $2 \mathrm{~h}$ & - & - \\
\hline $2 \mathrm{i}$ & - & 512 \\
\hline $2 j$ & 128 & 1024 \\
\hline $2 \mathrm{k}$ & 128 & 512 \\
\hline 21 & - & 512 \\
\hline $2 m$ & 128 & - \\
\hline $2 n$ & - & - \\
\hline 20 & 128 & - \\
\hline $2 r$ & 256 & 1024 \\
\hline $2 s$ & - & 1024 \\
\hline $2 t$ & 64 & 512 \\
\hline $2 u$ & 64 & - \\
\hline $2 v$ & 128 & - \\
\hline $2 w$ & - & - \\
\hline $2 x$ & - & - \\
\hline 5-Fluorocytosine & 10 & NT \\
\hline Miconazole & NT & 50 \\
\hline
\end{tabular}

- No inhibition

NT - Not tested

a Solvent: DMSO

ze of the cycloalkyl ring helps increase the capacity to inhibit cell growth. Cycloheptyl derivatives $\mathbf{2 r}, \mathbf{2 n}, \mathbf{2} \mathbf{0}$ and $\mathbf{2 x}$ were thus the most active compounds, while all cyclopenthyl derivatives were inactive compounds.

The antiproliferative activity of the compounds was greater against the HEP cells compared to the other two cell lines, as can be seen in compounds $\mathbf{2} \mathbf{i}, \mathbf{2 n}, \mathbf{2} \mathbf{o}$ and $\mathbf{2 x}$ with inhibition of $95.4,57.3,71.0$ and $52.6 \%$, respectively. The most active cycloalkyl[ $b]$ thiophene compound identified in this study was 2 r, which inhibited the growth of HT29, NCI H-292 and HEP cancer cell lines by 100.0, 100.0 and $97.5 \%$, respectively. 
B. C. C. Souza et al.: Preliminary antifungal and cytotoxic evaluation of synthetic cycloalkyl[ $b]$ thiophene derivatives with PLS-DA analysis, Acta Pharm. 62 (2012) 221-236.

Cyclohepthyl $[b]$ thiophene derivatives also demonstrated that the size of the alkyl group in the para position of the benzylidene moiety seemed to be important for antiproliferative activity. In this context, a comparison of substituent effects revealed that the replacement of $i$-propyl (2o) by an ethyl (2n) group resulted in a slight reduction in antitumor activity (reduction of 71.0 to $57.3 \%$ for the HEP cell, and of 45.7 to $42.5 \%$ for the NCI H-292 cell), and the replacement of ethyl (2n) by a methyl (2m) group resulted in a substantial loss of activity (reduction of 57.3 to $22.1 \%$ for the HEP cell, 42.5 to $25.8 \%$ for the NCI H-292 cell and 61.6 to $11.0 \%$ for the HT29 cell).

Another structural feature that can be associated with complete loss of activity in these derivatives is the presence of bulky or large arylidenes linked to the 2-amino position, as can be seen in compounds which have a quinoline moiety $(2 \mathbf{s}, \mathbf{2 t}$ and $\mathbf{2} \mathbf{u})$, and in compounds $\mathbf{2 k}, \mathbf{2} \mathbf{p}$ and $\mathbf{2 q}$, which represent 4-benzyloxy-benzylidene, 4-morpholinyl-benzylidene and 4-pyrrolidinyl-benzylidene, respectively.

\section{Computational methods}

The goal of PLS regression is to provide a dimension reduction strategy in a situation where we want to relate a set of response variables $Y$ to a set of predictor variables $X$. The PLS-DA is a PLS regression where $Y$ is a set of binary variables describing the effects of a category variable on $X$; i.e., the number of dependent, or response, variables is equal to the number of categories. The best way to extract information from the PLS-DA is graphically, by plotting the obtained matrices.

Table V. Antiproliferative activity of the synthesized compounds

\begin{tabular}{|c|c|c|c|}
\hline \multirow{2}{*}{ Compd. ${ }^{a}$} & \multicolumn{3}{|c|}{ Inhibition $(\%)^{b, c}$} \\
\hline & HT29 & NCI H-292 & HEP \\
\hline $2 b$ & $11.1 \pm 2.7$ & $30.4 \pm 1.1$ & $20.8 \pm 6.4$ \\
\hline $2 d$ & $24.9 \pm 3.8$ & $24.2 \pm 5.5$ & $1.6 \pm 1.0$ \\
\hline $2 e$ & $0.0 \pm 0.0$ & $29.5 \pm 3.4$ & $0.0 \pm 3.1$ \\
\hline $2 \mathrm{~h}$ & $11.3 \pm 4.8$ & $23.5 \pm 1.2$ & $11.3 \pm 0.4$ \\
\hline $2 \mathbf{i}$ & $53.7 \pm 1.9$ & $50.9 \pm 4.0$ & $95.4 \pm 0.0$ \\
\hline $2 j$ & $4.9 \pm 0.6$ & $18.4 \pm 0.2$ & $3.7 \pm 4.8$ \\
\hline $2 \mathrm{~m}$ & $11.0 \pm 0.6$ & $25.8 \pm 1.1$ & $22.1 \pm 0.5$ \\
\hline $2 n$ & $61.6 \pm 2.0$ & $42.5 \pm 2.7$ & $57.3 \pm 5.0$ \\
\hline 20 & $28.9 \pm 1,5$ & $45.7 \pm 3.3$ & $71.0 \pm 3.9$ \\
\hline $2 r$ & $100.0 \pm 0.5$ & $100.0 \pm 0.0$ & $97.5 \pm 2.5$ \\
\hline $2 w$ & $34.7 \pm 0.7$ & $33.9 \pm 3.8$ & $24.1 \pm 2.7$ \\
\hline $2 x$ & $25.0 \pm 2.0$ & $27.9 \pm 2.7$ & $52.6 \pm 2.9$ \\
\hline
\end{tabular}

a Solvent: DMSO

b $25 \mu \mathrm{g} \mathrm{mL}^{-1}$

c Mean $\pm \mathrm{SD}, n=3$. 
B. C. C. Souza et al.: Preliminary antifungal and cytotoxic evaluation of synthetic cycloalkyl[ $b]$ thiophene derivatives with PLS-DA analysis, Acta Pharm. 62 (2012) 221-236.

PLS-DA was applied in this study to improve the results obtained by the classic image analysis and to identify the significant spots responsible for the differences in antiproliferative activity against the three cell lines, which were similar to each other. The models presented the following statistical indices:

$-R^{2} \sim 0.80$ - coefficient of determination, the squared multiple correlation coefficient, which is the total variance of the response explained by a regression model;

- SDEP 0.70 - standard deviation error of prediction, also known as standard error in prediction SEP or PSE, which is the function of the predictive residual sum of squares;

- $Q^{2} \sim 0.60$ - predictive ability of the model.

A large difference was observed between active (triangle up) and inactive (triangle down) compounds for the three cell lines (Fig. 1). This graph plots $X$-scores (T) against $Y$-scores (U). It provides a clear idea of the correlation between the $X^{\prime}$ s and the $Y^{\prime}$ s obtained in the model for each of the LVs. LVs are linear combinations of the original $X$ -variables.

The variables highlighted in the studies with the three cell lines differ only by Angstroms but showed that hydrogen bond acceptor regions were characteristic of the active compounds. The plot weight, obtained in the study with the NCI H-292 cell line, reflects the behavior observed in all investigations (Fig. 2). Active compounds showed greater interactions between the probes DRY-DRY, N1-N1 and DRY-N1.

The most active compound (2r) was the only one that exhibited an O-N1 interaction (represented by the variable VAR246) (triangle up). In Fig. 3 this variable corresponds to an interaction between 4.40 and $4.80 \AA$, and characterizes a hydrogen bond acceptor region.

a)

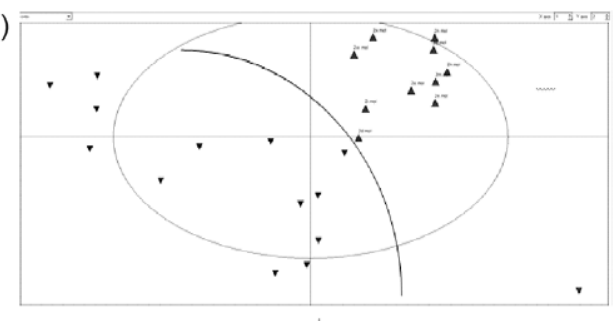

c)

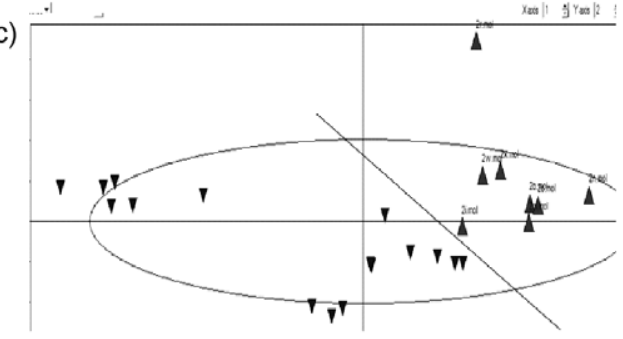

b)

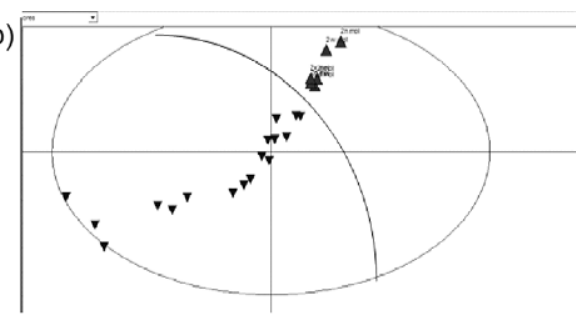

Fig. 1. Plot scores obtained from PLS-DA, distribution and separation of the set by the best model. Active (triangle up) and inactive (triangle down) compounds against the investigated cell lines: a) HT 29 cell line, b) NCI H-292 cell line, and c) HEP cell line. 
B. C. C. Souza et al.: Preliminary antifungal and cytotoxic evaluation of synthetic cycloalkyl[b]thiophene derivatives with PLS-DA analysis, Acta Pharm. 62 (2012) 221-236.

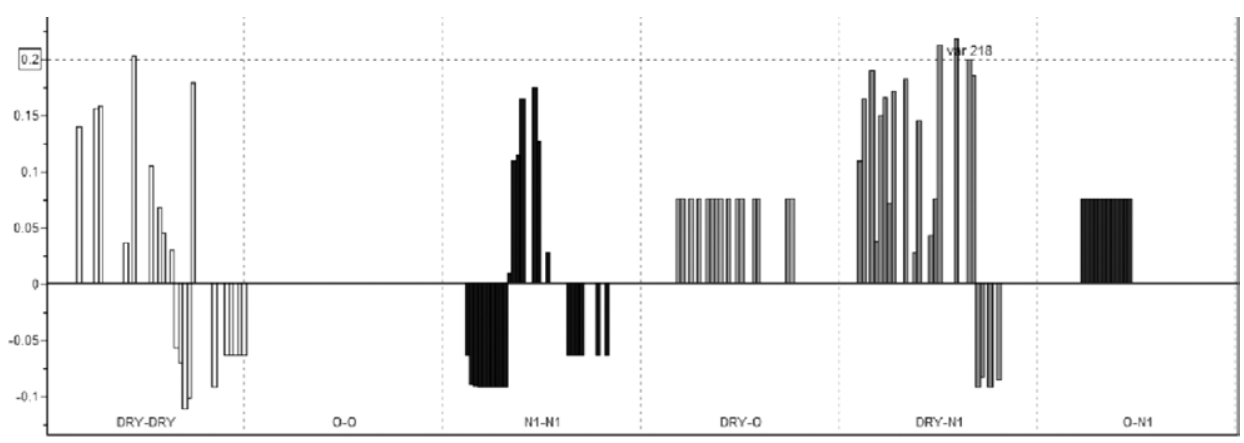

Fig. 2. Plot weight obtained with the NCI H-292 cell line, selecting the active compounds. Greater intensity and number of positive influence of the variables are generated on the interaction of probes DRY-DRY, N1-N1 and DRY-N1 in active compounds.

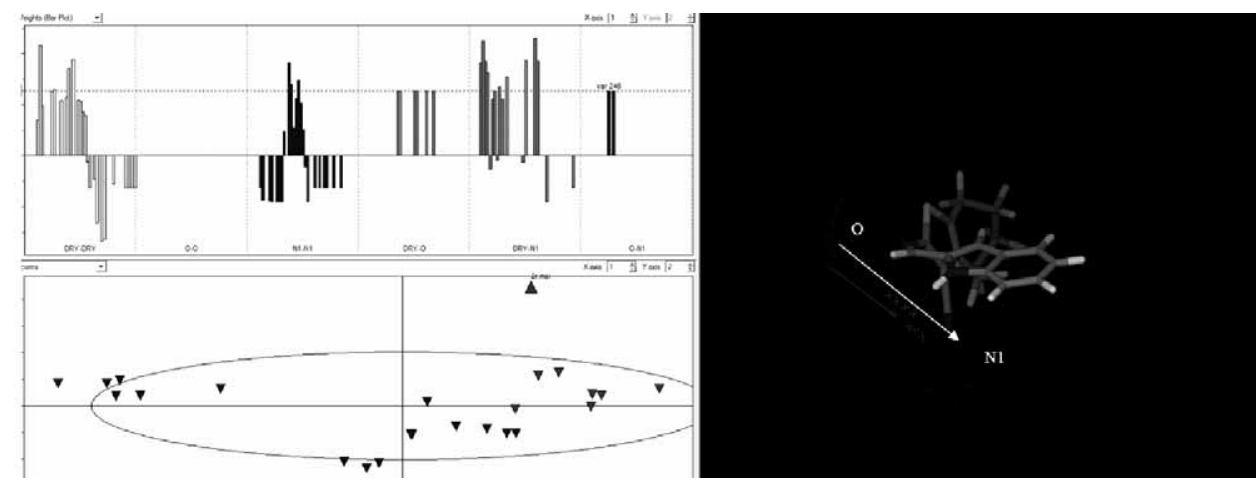

Fig. 3. Disclosure of O-N1 interaction, VAR 246, in the most active compound (2r) - (triangle up).

\section{CONCLUSIONS}

In short, novel thiophene derivatives substituted with an arylidene moiety were synthesized, characterized on the basis of their physical, analytical, and spectral data, and preliminarily evaluated for their in vitro antifungal and antiproliferative properties.

Antifungal studies revealed that the results for the antifungal activity of cycloalkyl$[b]$ thiophene derivatives ranged from excellent to moderate and that the most promising compounds were those that had a cyclohexyl ring linked to the thiophene ring $(\mathbf{2} \mathbf{d}, \mathbf{2} \mathbf{g}$, $2 \mathbf{i}-\mathbf{k}$ and $2 \mathbf{t}$ ).

The results for antiproliferative activity indicate that the most active compounds are cycloheptyl[ $b]$ thiophene derivatives, especially 2-[(1H-indol-2-yl-methylidene $)$ amino $]-$ 5,6,7,8-tetrahydro-4H-cyclohepta[ $b]$ thiophene-3-carbonitrile (2r). 
B. C. C. Souza et al.: Preliminary antifungal and cytotoxic evaluation of synthetic cycloalkyl[b]thiophene derivatives with PLS-DA analysis, Acta Pharm. 62 (2012) 221-236.

The chemometric tool (PLS-DA) applied in this study generated good exploratory and predictive results. The behavior of the binary free PLS-DAwas similar for the three human cancer cell lines studied. The descriptors having shape characteristics were strongly correlated with the biological data. Higher weight variables highlight the interactions between the DRY-DRY N1-N1, DRY-N1 probes. Hydrogen bond acceptor regions are characteristic of active compounds and the presence of $\mathrm{O}-\mathrm{N} 1$ interaction, as found in 2r, increased the antitumor activity.

Finally, it can be concluded that cycloalkyl $[b]$ thiophene derivatives could be considered promising compounds for the discovery of new antitumor agents. Further investigation of the mode of action at molecular level needs to be carried out, and more extensive studies are needed to determine additional physicochemical and biological parameters in order to provide a deeper insight into the SAR and to optimize the efficacy and safety of this series of compounds.

Acknowledgements. - We are indebted to the Conselho Nacional de Desenvolvimento Científico e Tecnológico (CNPq) and UEPB, through the Programa de Incentivo à Pós-Graduação e Pesquisa/ PRPGP for financial support. The authors thank the CNPQ and the Fundação de Amparo à Ciência e Tecnologia do Estado de Pernambuco (FACEPE) for scholarships of Luciana Scottiand Beatriz C. C. Souza.

\section{REFERENCES}

1. Z. Puterová, A. Krutošíková and D. Végh, Gewalg reactions: synthesis, properties and applications of substituted 2-aminothiophenes, Arkivoc i (2010) 209-246.

2. K. Balamurugan, S. Perumal, A. S. K. Reddy, P. Yogeeswari and D. Sriram, A facile domino protocol for the regioselective and discovery of novel 2-amino-5-arylthieno-[2,3-b]thiophenes as antimycobacterial agents, Tetrahedron Lett. 50 (2009) 6191-6195; DOI: 10.1016/j.tetlet.2009.08.085.

3. I. M. I. Fakhr, M. A. A. Radwan, S. El-Batran, M. E. Omar, A. El-Salam and S. M. El-Shenawy, Synthesis and pharmacological evaluation of 2-substituted benzo[ $b]$ thiophenes as anti-inflammatory and analgesic agents, Eur. J. Med. Chem. 44 (2009) 1718-1725; DOI: 10.1016/j.ejmech.2008. 02.034 .

4. A. M. Isloor, B. Kalluraya and K. S. Pai, Synthesis, characterization and biological activities of some new benzo[b]thiophene derivatives, Eur. J. Med. Chem. 45 (2010) 825-830; DOI: 10.1016/j. ejmech.2009.11.015.

5. A. E. G. E. Amr, M. H. Sherif, M. G. Assy, M. A. Al-Omar and I. Raga, Antiarrhythmic, serotonin antagonist and antianxiety activities of novel substituted thiophene derivatives synthesized from 2-amino-4,5,6,7-tetrahydro- $N$-phenylbenzo[b]thiophene-3-carboxamide, Eur. J. Med. Chem. 45 (2010) 5935-5942; DOI: 10.1016/j.ejmech.2010.09.059.

6. M. Gütschow, L. Kuerschner, U. Neumann, M. Pietsch, R. Löser, N. Koglin and K. Eger, 2-(Diethylamino)thieno[1,3] oxazin-4-ones as stable inhibitors of human leukocyte elastaze, J. Med. Chem. 42 (1999) 5437-5447; DOI: 10.1021/jm991108w.

7. A. B. Pinkerton, T. T. Lee, T. Z. Hoffman, Y. Wang, M. Kahraman, T. G. Cook, D. Severance, T. C. Gahman, S. A. Noble, A. K. Shiau and R. I. Davis, Synthesis and SAR of thiophene containing kinesin spindle protein (KSP) inhibitors, Bioorg. Med. Chem. Lett. 17 (2007) 3562-3569; DOI: 10.1016/j.bmcl.2007.04.076.

8. L. Aurelio, C. Valant, H. Figler, B. L. Flynn, J. Linden, P. M. Sexton, A. Christopoulos and P. J. Scammells, 3- and 6-Substituted 2-amino-4,5,6,7-tethahydrothieno[2,3-c]pyridines as A1 adeno- 
B. C. C. Souza et al.: Preliminary antifungal and cytotoxic evaluation of synthetic cycloalkyl[ $b]$ thiophene derivatives with PLS-DA analysis, Acta Pharm. 62 (2012) 221-236.

sine receptor allosteric modulators and antagonists, Bioorg. Med. Chem. 17 (2009) 7353-7361; DOI: $10.1016 /$ j.bmc.2009.08.024.

9. F. J. B. Mendonça Jr., R. G. Lima-Neto, T. B. Oliveira, M. C. A. Lima, I. R. Pitta, S. L. Galdino, R. M. D. Cruz, R. S. A. Araújo and R. P. Neves, Synthesis and evaluation of the antifungal activity of 2-(substituted-amino)-4,5-dialkyl-thiophene-3-carbonitrile derivatives, Lat. Am. J. Pharm. 30 (2011) 1492-1499.

10. A. A. Abu-Hashem, M. F. El-Shehry and F. A. Badria, Design and synthesis of novel thiophenecarbohydrazide, thienopyrazole and thienopyrimidine derivatives as antioxidant and antitumor agents, Acta Pharm. 60 (2010) 311-323; DOI: 10.2478/v10007-010-0030-y.

11. National Committee for Clinical and Laboratory Standards: Method M27-A2, NCCLS, Wayne (PA), Villanova, Pennsylvania, USA, 2002, pp. 1-29.

12. J. A. Barnett, R. W. Paire and D. Yarrow, Yeasts: Characteristics and Identification, Cambridge University Press, Cambridge 2000.

13. E. L. Souza, T. L. M. Stamford, E. O. Lima and V. N. Trajano, Effectiveness of Origanum vulgare L. essential oil to inhibit the growth of food spoiling yeasts, Food Control 18 (2007) 409-413; DOI: 10.1016/j.foodcont.2005.11.008.

14. P. J. Houghton, M. J. Howea, C. C. Lee and G. Steventon, Uses and abuses of in vitro tests in ethnopharmacology: visualizing an elephant, J. Ethnopharmacol. 110 (2007) 391-400; DOI: 10.1016/ j.jep.2007.01.032.

15. M. R. Boyd and K. D. Paull, Some practical considerations and applications of the national cancer institute in vitro anticancer discovery screen, Drug Develop. Res. 34 (1995) 91-109; DOI: 10.1002/ ddr.430340203.

16. M. Kožurková, D. Sabolová, L. Janovec, J. Mikeš, J. Koval, J. Ungvarský, M. Štefanišinová, P. Federočko, P. Kristian and J. Imrich, Cytotoxic activity of proflavinediureas: synthesis, antitumor, evaluation and DNA binding properties of 1',1"-(acridin-3,6-diyl)-3',3"-dialkyldiureas, Bioorg. Med. Chem.16 (2008) 3976-3984; DOI: 10.1016/j.bmc.2008.01.026.

17. N. L. Allinger, A hydrocarbon force-field utilizing V1 and V2 torsional terms, J. Am. Chem. Soc. 99 (1977) 8127-8134; DOI: 10.1021/ja00467a001.

18. M. J. S. E. Dewar, G. Zoebisch, E. F. Healy and J. J. P. Stewart, AM1: a new general purpose quantum mechanical molecular model, J. Am. Chem. Soc. 107 (1985) 3902-3909; DOI: 10.1021/ ja00299a024.

19. N. C. Cohen, Guidebook on Molecular Modeling, in Drug Design, Academic Press, San Diego 1996, p. 361.

20. http://www.moldiscovery.com/soft_pentacle.php, access date 4/12/2012.

21. C. A. Boateng, S. V. K. Eyunni, X, Y. Zhu, J. R. Etukala, B. A. Bricker, M. K. Ashfaq, M. R. Jacob, S. I. Khan, L. A. Walker and S. Y. Ablordeppey, Synthesis and evaluation against opportunistic fungal pathogens, Bioorg. Med. Chem. 19 (2011) 458-470; DOI: 10.1016/j.bmc.2010.11.008. 
B. C. C. Souza et al.: Preliminary antifungal and cytotoxic evaluation of synthetic cycloalkyl[ $b]$ thiophene derivatives with PLS-DA analysis, Acta Pharm. 62 (2012) 221-236.

$S A \check{Z} E T A K$

\title{
Preliminarno ispitivanje antimikotskog i citotoksičnog djelovanja derivata cikloalkil[ $b]$ tiofena PLS-DA analizom
}

\author{
BEATRIZ C. C. SOUZA, TIAGO B. DE OlIVEIRA, THIAGO M. AQUINO, MARIA C. A. DE LIMA, IVAN R. PITTA, \\ SUELY L. GALDINO, EDELTRUDES O. LIMA, TERESINHA GONÇALVES-SILVA, GARDÊNIA C. G. MILITÃO, \\ LUCIANA SCOTTI, MARCUS T. SCOTTI i FRANCISCO J. B. MENDONÇA Jr.
}

Koristeći supstituirane aromatske aldehide u Gewaldovim aduktima 1a-c sintetizirani su derivati 2-[(ariliden)amino]-cikloalkil[ $b]$ tiofen-3-karbonitrila (2a-x). Ispitano je antimikotsko djelovanje tih spojeva na gljivice Candida krusei i Criptococcus neoformans te antiproliferativno djelovanje na tri humane tumorske stanične linije (HT29, NCI H-292 i HEP). Za antiproliferativno djelovanje primijenjena je metoda parcijalnih najmanjih kvadrata (PLS) koristeći softverski program Pentacle. Neki od ispitanih spojeva pokazuju obećavajuće antimikotsko i antiproliferativno djelovanje. Najjače antimikotsko djelovanje imaju cikloheksil[ $b]$ tiofen derivati, a najjače antiproliferativno djelovanje cikloheptil[ $b]$ tiofen derivati, posebice 2-[(1H-indol-2-il-metiliden)amino]-5,6,7,8-tetrahidro-4H-ciklohepta[ $b]$ tiofen-3-karbonitril (2r), koji inhibira više od 97 \% rast svih triju ispitivanih staničnih linija. Primijenjena PLS diskriminirajuća analiza dala je dobre istraživačke i prognostičke rezultate i pokazala da deskriptori dobro koreliraju s biološkim rezultatima.

Ključne riječi: cikloalkil[b]tiofen derivati, antimikotsko djelovanje, antiproliferativno djelovanje, PLS-DA metoda, program Pentacle

Departamento de Antibióticos, Universidade Federal de Pernambuco, Recife-PE 50670-910, Brazil

Departamento de Ciências Farmacêuticas, Universidade Federal da Paraíba, João Pessoa-PB, 58051-970, Brazil

Departamento de Engenharia e Meio Ambiente, Universidade Federal da Paraíba, Campus IV; Rio Tinto-PB 58297-000, Brazil

Departamento de Ciências Biológicas, Universidade Estadual da Paraíba, CCBSA, João Pessoa-PB 58070-450, Brazil 